\title{
Cosmology with dark energy decaying through its chemical-potential contribution
}

\author{
J Besprosvany \\ Instituto de Física, Universidad Nacional Autónoma de México, Apartado Postal \\ 20-364, México 01000, Distrito Federal, México \\ E-mail: bespro@fisica.unam.mx
}

\begin{abstract}
.
The consideration of dark energy's quanta, required also by thermodynamics, introduces its chemical potential into the cosmological equations. Isolating its main contribution, we obtain solutions with dark energy decaying to matter or radiation. When dominant, their energy densities tend asymptotically to a constant ratio, explaining today's dark energy-dark matter coincidence, and in agreement with supernova redshift data.

PACS numbers: 98.80.-k, 98.80.Es, 98.80.Bp
\end{abstract}

Keywords: dark energy, chemical potential, decay

Reference: J. Phys. A: Math. Theor. 40 7099-7104 (2007) doi: 10.1088/1751$8113 / 40 / 25 /$ S68

\section{Introduction}

Dark energy is a component of the universe whose negative pressure, characteristic of the quantum vacuum, accelerates its expansion. Evidence for its existence has recently accumulated from independent sources as the supernova redshift far-distance relation [1], 2], structure formation [3], the microwave background radiation [4, and lensing [5].

The cosmological constant $\Lambda$, dark-energy's original conception, was added by Einstein in the application of general relativity to cosmology in 1917 in order to describe a static universe [6], building on a 1890s proposal by Neumann and Seeliger, who introduced it in a Newtonian framework for the same reasons. Its contribution in the Einstein equations

$$
R_{\mu \nu}-\frac{1}{2} g_{\mu \nu} R-\Lambda g_{\mu \nu}=8 \pi T_{\mu \nu}
$$

equilibrates gravity's attraction in a matter universe; here $R_{\mu \nu}$ is the Ricci tensor, $g_{\mu \nu}$ the metric tensor, which describe the geometry, and $T_{\mu \nu}$ is the energy-momentum 
tensor; we use units with the Newton, Planck, Boltzmann, and light-speed constants $G=\hbar=k_{B}=c=1$, except when given explicitly, as needed.

Zeld'ovich sought to connect it to the quantum vacuum[7]. This requires its reinterpretation as a $T_{\mu \nu}$ component in Eq. 1. The vacuum energy density of particle fields with mass $m \ll M_{P}=\frac{1}{\sqrt{G}}$ is obtained by summing over its modes $\mathbf{k}$ :

$$
\rho_{\Lambda P}=\frac{1}{(2 \pi)^{3}} \int^{M_{P}} d^{3} k \sqrt{k^{2}+m^{2}} \simeq 3 \times 10^{114} \frac{\mathrm{GeV}}{\mathrm{cm}^{3}} ;
$$

the natural cutoff is the Planck-mass scale $M_{P}$, the only possible mass conformed of $G, \hbar$, and $c$, while in today's universe $\rho_{\Lambda 0} \simeq 4 \times 10^{-6} \mathrm{GeV} / \mathrm{cm}^{3} \cdot \rho_{\Lambda 0}$ represents $\Omega_{\Lambda 0}=\rho_{\Lambda 0} / \rho_{c 0} \simeq .73$ of its critical energy density $\rho_{c 0}$ today [8], and in a flat universe [9] $\sum \Omega_{i}=1$. The rest corresponds mainly to matter, dark and baryonic, the latter conforming $\Omega_{b 0} \simeq .044$ only[8]. Dark energy's origin, its smallness by 122 orders of magnitude with respect to the vacuum's natural Planck scale, and the coincidence of its present energy-density scale with the universe's remain puzzling; dynamic behavior points to a possible explanation.

The energy components are generally perfect fluids, described by their energy tensor $T_{\nu}^{\mu(i)}=\left(\rho_{i}, p_{i}, p_{i}, p_{i}\right)$ (at rest), with $T_{\mu \nu}=\sum_{i} T_{\mu \nu}^{(i)}$. Radiation and matter are characterized by an equation of state

$$
p_{i}=w_{i} \rho_{i},
$$

where $w_{r}=1 / 3$ for radiation (and for relativistic Fermi or Bose gases,) and $w_{m}=0$ for non-relativistic matter. Under the isotropic Robertson-Walker metric $d s^{2}=d t^{2}-$ $R^{2}(t)\left(d x^{2}+d y^{2}+d z^{2}\right)$, Eq. 1 implies the Friedmann equation

$$
H^{2}=\frac{8 \pi}{3} \rho_{c}=\frac{8 \pi}{3}\left(\rho_{\Lambda}+\rho_{r}+\rho_{m}\right)
$$

where $x, y, z$ are commoving Cartesian coordinates, $R$ is the scale factor, depending on time $t$, as do $\rho_{i}$, and $H=\dot{R} / R$, the Hubble parameter (a dot denotes time derivative.)

The energy-conservation equation within an expanding volume $V \sim R^{3}$

$$
\sum_{i} d\left(\rho_{i} V\right)=-\sum_{i} p_{i} d V
$$

is implied by the contraction of Eq. 1. When decoupled, each contribution also satisfies

$$
d\left(\rho_{i} V\right)=-p_{i} d V
$$

Eq. 6] can also be interpreted as a particular case of the first law of thermodynamics

$$
d(\rho V)=-p d V+\mu d N+T d S,
$$

with additional contributions from the entropy $S$, and the particle number $N$, where $T$ is the temperature and $\mu$ the chemical potential. When non-interactive, radiation has $\mu=0$, baryonic matter is conserved, $d N=0$, and for both $d S=0$. These conditions may not be true for dark energy or dark matter. In this paper, we show that the consideration of dark-energy's quanta modifies the cosmological equations through the $\mu d N$ term in Eq. 7, with the implication that dark energy decays to another component. 
Thus, the derived asymptotic energy-density constant ratio of the dominant components reproduces the coincidence of dark energy and dark matter today. The entropy term $T d S$ in Eq. 7 will be neglected, as dark energy is associated to low-energy states. We first classify the chemical potential associated to the pressure in Eq. 3 (Section 2.) Relating it to a decay width, we consider its main contribution to the cosmological equations, which are exactly solved for two components, and we then apply the model to the supernova data (Section 3.) We finally draw conclusions (Section 4.)

\section{Dark-energy's equation of state}

The form of Eq. 2 implies $\Lambda$ generates a pressure $p_{\Lambda}=-\rho_{\Lambda}$, so $w_{\Lambda}=-1$ for the vacuum energy. The parametric extension to arbitrary negative values $w_{\Lambda}$, following Eq. 3, with similar properties [10], [11, suits the lack of precise knowledge about it. Whatever is its nature, and with a name not bound to its constancy, dark energy should contain quanta[7], as any other form of energy in the universe, and so, the energy dependence on its number $N$ should be accounted for. Within the relation

$$
E=c V^{-w},
$$

consistent with Eq. 3, $c$ a constant, if the energy dependence remains extensive, another such quantity is required. Using $N$ for such a variable,

$$
E=c^{\prime} N\left(\frac{V}{N}\right)^{-w}
$$

introduces an $N$-dependence, with $c^{\prime}$ an (intensive) constant, except in the $w=-1$ case, in agreement with the view that no quanta are associated to the vacuum.

Eq. 9, also consistent with Eq. 3, implies the contribution

$$
n \mu=(1+w) \rho,
$$

where $n=N / V$ is the particle density.

We concentrate on dark energy satisfying Eq. 3. Using the thermodynamic relation

$$
s=\frac{1}{T}(\rho+p-n \mu),
$$

with $s=S / V$ the entropy density, we identify two limiting cases: (1) in the zero-entropy regime $(s=0)$,

$$
\rho_{\Lambda w}=c_{w} n^{1+w}
$$

with $c_{w}$ a constant, and $n \mu_{\Lambda w}=(1+w) \rho$, as for Eq. 10; (2) the radiation-like assumption, $\mu_{r w}=0$, leads to

$$
s_{r w}=c_{r w} \rho^{\frac{1}{1+w}}
$$

$\left(c_{r w}\right.$ a constant.)

Case (1) with Eq. 10, induced from Eq. 3, or Case (2) with $\mu_{r w}=0$ represent special conditions; similarly to Eq. 3, the most general linear $\rho$-dependence for the chemical potential requires the new parameter $\chi$ in

$$
n \mu_{w \chi}=(1+w+\chi) \rho .
$$


Eqs. 3, 11, and 14 then generally lead to $s_{w \chi}=n\left(\frac{\rho}{c_{w} n^{1+w}}\right)^{-\frac{1}{\chi}}$. From the resulting temperature $T_{w \chi}=-\frac{\chi \rho}{n}\left(\frac{\rho}{c_{w} n^{1+w}}\right)^{\frac{1}{\chi}}$, it follows that $\chi \neq 0$ signals a non-zero $T_{w \chi}$. In fact, $s_{w \chi}$ contains the $s=0$ limit, as Eq. 12 is approached with $\rho \sim \rho_{\Lambda w}$ for $\chi \rightarrow 0$, and for the $\mu_{r w}=0$ case in Eq. 13, $s_{w \chi}=s_{r w}$ for $\chi=-w-1$, and $c_{r w}=c_{w}^{1 / \chi}$. The knowledge of $w_{\Lambda}$, and these limits suggest $\chi$ is also $\mathrm{O}(1)$.

The modification of Eq. 6 by the chemical-potential contribution is analyzed next.

\section{Cosmological equations with dark energy's chemical potential}

The chemical potential can be written as

$$
\mu_{\Lambda} d N=\mu_{\Lambda}\left(n_{\Lambda} d V+V d n_{\Lambda}\right)
$$

changes in particle numbers through decay are associated to partial widths $\Gamma$, and, ultimately, to interactions. In the universe's evolution in $d t$, we distinguish the two contributions: (1) $N \Gamma_{1} d t=n_{\Lambda} \mu_{\Lambda} d V=\left(1+w_{\Lambda}+\chi\right) \rho_{\Lambda} d V$ is associated with decay due to its expansion

$$
n_{\Lambda} \Gamma_{1}=3\left(1+w_{\Lambda}+\chi\right) H \rho_{\Lambda} \sim \rho_{\Lambda}^{3 / 2},
$$

given $H \sim \rho_{\Lambda}^{1 / 2}$; (2) $N \Gamma_{2} d t=\mu_{\Lambda} V d n_{\Lambda}$ contains terms that are not of this form; it could account for any other out-of-equilibrium conceivable decay process linked to interactions. For the gravitational interaction, and $T_{w \chi}=0, \Gamma_{2} \sim \sigma n_{\Lambda} v \sim\left(1 / M_{P}^{4}\right) n_{\Lambda} \rho_{\Lambda}^{1 / 2}$, where for the cross section $\sigma \sim\left(1 / M_{P}^{4}\right) \rho_{\Lambda}^{1 / 2}$, given a tree-level gravitational interaction, and the dimensionally fit power of the only relevant variable $\rho_{\Lambda}$; the velocity $v \sim c=1$, so $n_{\Lambda} \Gamma_{2} \sim \rho_{\Lambda}^{\frac{2}{w_{\Lambda}+1}+1 / 2}$, using $\rho_{\Lambda w}$ in Eq. 12, Comparing with $n_{\Lambda} \Gamma_{1} \sim \rho_{\Lambda}^{3 / 2}$, from Eq. 16, for $-1<w_{\Lambda}<1, \Gamma_{2} \ll \Gamma_{1}$ as $\rho_{\Lambda} \rightarrow 0$. Similarly, this will always occur for low $T_{w \chi} \neq 0$, implying still $\rho_{\Lambda} \sim \rho_{\Lambda w}$, but high enough for the thermic contribution to be dominant so [12] $\sigma \sim\left(1 / M_{P}^{4}\right) T_{w \chi}^{2}$. Another type of interaction can be dominant for some time, but it will eventually be overridden by the $\Gamma_{1}$ term. Lower powers of $\rho_{\Lambda}$, e. g., a constant decay rate $n_{\Lambda} \Gamma_{2} \sim \rho_{\Lambda}$, could make a significant cosmological contribution, but it would have to be fine-tuned to give the present parameters[13]. Thus, the $\Gamma_{2}$ term can and will be neglected.

Under such circumstances, we use changes of the form $\partial N_{\Lambda} / \partial V=n_{\Lambda}$ in Eq. 15. We obtain, using Eqs. 7, 16,

$$
\dot{\rho}_{\Lambda}+3\left(w_{\Lambda}+1\right) H \rho_{\Lambda}=3\left[\left(w_{\Lambda}+1\right)+\chi\right] H \rho_{\Lambda} .
$$

Energy conservation in Eq. 5 demands that energy be transferred, which we assume occurs for only another dominant $i$ component in Eq. 4,

$$
\dot{\rho}_{i}+3\left(w_{i}+1\right) H \rho_{i}=-3\left[\left(w_{\Lambda}+1\right)+\chi\right] H \rho_{\Lambda} .
$$

The set of Eqs. 4, 17, 18 describes a two-fluid system with $\rho_{\Lambda}$ decaying out of equilibrium

as is common in many universe processes [12]. No energy transfer is produced for $w_{\Lambda}+1+\chi=0$, that is, for the radiation-like case with $n \mu_{w \chi}=0$ in Eq. 14. We also find (see Eq. 17) dark-energy decay for $\chi<0$. A decaying cosmological constant was first 
conceived by Bronstein[14] to explain the universe's time direction, and recent study starts with Ref. [15], with various phenomenological decay laws then considered[16]; quintessence models with a similar energy interchange have also been studied[17]. By substituting $H$ in Eq. 4 into Eq. 17, we obtain

$$
\rho_{i}=-\rho_{\Lambda}+\frac{\dot{\rho}_{\Lambda}^{2}}{24 \pi \chi^{2} \rho_{\Lambda}^{2}} .
$$

Substituting this into Eq. 18, we get

$$
6 \chi \rho_{\Lambda} \ddot{\rho}_{\Lambda}+\left(d_{i}-6 \chi\right) \dot{\rho}_{\Lambda}^{2}-24 \pi\left[d_{i}-3\left(1+w_{\Lambda}\right)\right] \chi^{2} \rho_{\Lambda}^{3}=0,
$$

where $d_{i}=3\left(w_{i}+1\right)$. $t$ as inverse function of $\rho_{\Lambda}$ can be integrated, where initially $\rho_{\Lambda_{i}}$ at $t_{i}$

$$
t-t_{i}=\int_{\rho_{\Lambda}}^{\rho_{\Lambda_{i}}} d \rho\left(\frac{d_{i}+3 \chi}{24 \chi^{2} \pi\left[d_{i}-3\left(w_{\Lambda}+1\right)\right] \rho^{3}+3\left(d_{i}+3 \chi\right) \chi C \rho^{2-\frac{d_{i}}{3 \chi}}}\right)^{\frac{1}{2}}
$$

$C$ accounts for initial conditions for $\rho_{i}$, and we have chosen the solution for which $R$ increases and $\rho_{\Lambda}$ decreases. For some $\chi, w_{\Lambda}, t\left(\rho_{\Lambda}\right)$ can be given explicitly in terms of hypergeometric and elliptic functions. Using Eqs. 19, 21] one finds

$$
\rho_{c} \approx \frac{24 \chi^{2} \pi\left[d_{i}-3\left(w_{\Lambda}+1\right)\right] \rho_{\Lambda}+\left(d_{i}+3 \chi\right) 3 \chi C \rho_{\Lambda}{ }^{-\frac{d_{i}}{3 \chi}}}{24 \pi \chi^{2}\left(d_{i}+3 \chi\right)} .
$$

One derives that for $-d_{i} / 3<\chi<0$

$$
\lim _{\rho_{\Lambda} \rightarrow 0} \frac{\rho_{\Lambda}}{\rho_{c}}=\frac{d_{i}+3 \chi}{d_{i}-3\left(w_{\Lambda}+1\right)}
$$

within the wide set of initial conditions $C \ll \rho_{\Lambda 0}^{1+\frac{d_{i}}{3 \chi}}$, so $\Omega_{i}$ and $\Omega_{\Lambda}$ will acquire a fixed asymptotic value.

Such an asymptotic behavior fits the supernova data[18] interpreted under Eq. 23, with dark matter and dark energy evolving with a constant ratio. Considering baryonic matter, dark matter and dark energy (the latter two evolving as $R^{3 \chi_{0}}$ ), assuming asymptotic behavior sets in as early as $z=2$, with the constant $\chi_{0}=-.48$, and as shown in Fig. 1 (and compared with the fitting non-asymptotic model, and non-fitting $\Omega_{\Lambda 0}=0$, and cosmological-constant $\Omega_{\Lambda 0}=1$ cases), one can reproduce the luminosity distance $d_{L}=H_{0}^{-1}(1+z) \int_{0}^{z} d z^{\prime}\left[\Omega_{b 0}\left(1+z^{\prime}\right)^{3}+\left(1-\Omega_{b 0}\right)\left(1+z^{\prime}\right)^{-3 \chi_{0}}\right]^{-1 / 2}$ up to the measured redshift $z \sim 2$. We note that the fit is independent of $\Omega_{\Lambda 0}$, as derives from the asymptotic regime. The choice of initial conditions ( $C$ in Eq. 21) sets the timing of the matter-dominated regime $\left(w_{i}=w_{m}=0\right.$ in Eq. 18) before the asymptotic one, to match the conventional cosmology.

\section{Conclusions}

In summary, account of dark energy's quanta allows for a dark-energy decaying model able to explain its coincidence with dark matter today, within classical general relativity and thermodynamics. It represents a departure from the zero-temperature 


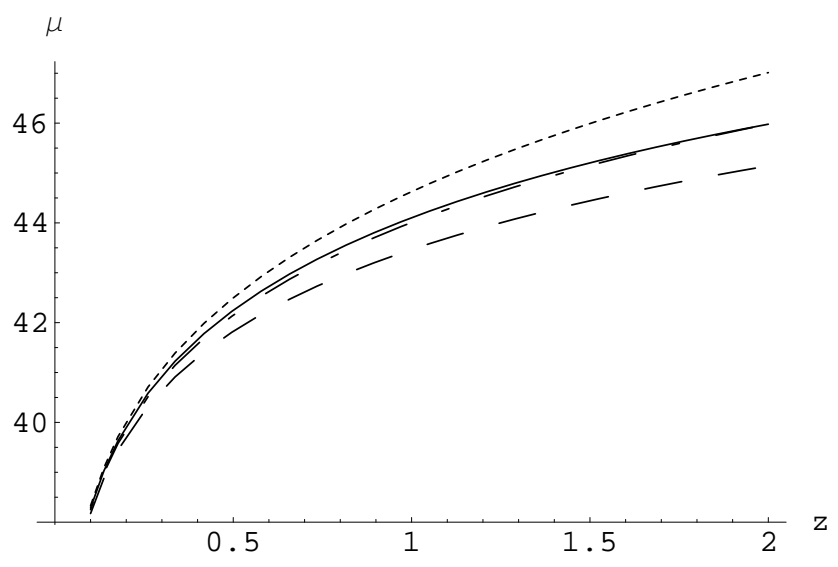

Figure 1. Comparison of magnitude $\mu=5 \log _{10}\left(d_{L} / \mathrm{Mpc}\right)+25$ of luminosity distance $d_{L}$, as a function of redshift $z$, for flat models. For non-asymptotic models with $w_{\Lambda}=-1$, and (a) $\Omega_{m 0}=0, \Omega_{\Lambda 0}=1$ (dotted), (b) $\Omega_{m 0}=.27, \Omega_{\Lambda 0}=.73$ (line), and (c) $\Omega_{m 0}=1, \Omega_{\Lambda 0}=0$ (dashed); and (d) for asymptotic model with $\Omega_{b 0}=.044$, and $\chi_{0}=-.48$ (dot-dashed). The reduced Hubble parameter $h=.71$ was used for all cases.

cosmological constant, while it maintains the results of the standard cosmology. This supports a conservative approach in which known physical elements can provide new information [19]. Dark energy's coincidence with the critical density today is connected to the universe evolution, in which events occur by contingency, rather than chance. While microphysics [20] needs to elucidate the dark energy's equation of state, the universe already emerges as flat, interconnected, evolving deterministically, and in an inexorable process of accelerated expansion and decay.

\section{References}

[1] Perlmutter S et al 1998 Nature 39151

[2] Garnavich P M et al 1998 Astrophys. J. 50974

[3] Efstathiou G. Sutherland W J and Maddox S J 1990 Nature 348705

[4] Spergel D N et al 2003 Astrophys. J. Suppl. 148175

[5] Soucail G Kneib J P and Golse G 2004 Astron. Astrophys. 417 L33

[6] A. Einstein 1917 Sitzungsberichte der königliche Preussiche Akademie der Wissenschaften zu Berlin 142

[7] Zel'dovich Ya B 1967 JETP Lett. 6316 [ 1967 Pisma Zh. Eksp. Teor. Fiz. 6, 883 ]

[8] Eidelman S et al 2004 Phys. Lett. B 592

[9] Peebles P J E and Ratra B 2003 Rev. Mod. Phys. 75559

[10] Steinhardt P J 1997 in Critical Dialogues in Cosmology ed Turok N (Singapore: World Scientific )

[11] Turner M S and White M 1997 Phys. Rev. D 56 R4439

[12] Kolb E W and Turner M S 1994 The Early Universe (Reading, Mass: Adison-Wesley)

[13] Besprosvany J 2005, Cosmology from decaying dark energy, primoridal at the Planck scale Preprint astro-ph/0502439

[14] Bronstein M 1933 Phys. Z. Sowjetunion 373

[15] Özer M O and Taha M O 1987 Nucl. Phys. B 287776 
[16] Reuter M and Wetterich C 1987 Phys. Lett. B 188 38; Overduin J M and Cooperstock F I 1998 Phys. Rev. D 58 043506; Padmanabhan T 2003 Phys. Rep. 380235

[17] Amendola L Phys. Rev. D 62 (2000) 043511; Zimdahl W et al 2001 Phys. Lett. B 521133

[18] Riess A G et al 2004 Astrophys. J. 607665

[19] Besprosvany J 2004 Phys. Lett. B 578181

[20] Adler S L 1982 Rev. Mod. Phys. 54 729; Weinberg S 1989 Rev. Mod. Phys. 611

\section{Acknowledgement}

The author thanks A. de la Macorra and M. Giovannini for discussions, and acknowledges support from DGAPA-UNAM and HELEN. 